\title{
Regressão por mínimos quadrados parciais (PLS) e espectroscopia no UV/Vís para quantificação de corantes: agilidade e eficiência para estações de tratamentos de efluentes têxteis
}

Alan Rodrigues Teixeira Machado

alan.machado@prof.unibh.br

Adilson Cândido da Silva

adilsonufla@gmail.com

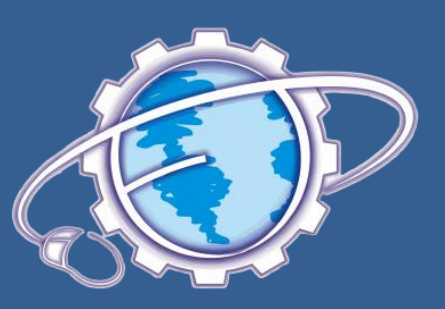

\section{RESUMO}

Os corantes são substâncias químicas com grande diversidade estrutural. Apresentam numerosas aplicações, com destaque para as indústrias: têxtil, alimentar e cerâmica. Várias são as técnicas analíticas utilizadas nestas indústrias para avaliação destes compostos. Entretanto, existe uma demanda por metodologias em que dois ou mais corantes sejam avaliados na mesma análise, tais metodologias quando utilizadas podem contribuir de forma significativa na redução dos custos, além de reduzir o tempo de análise e o consumo dos reagentes utilizados. Desta forma, objetivando a difusão de metodologias para quantificação simultânea de corantes, empregando a Regressão por Mínimos Quadrados Parciais (PLS), neste trabalho foi utilizada a espectroscopia no UV/Vís associada à PLS para construção de modelos para quantificação de três corantes: alaranjado de metila, azul de metileno e vermelho do congo. Os resultados permitem concluir que modelos de calibração, empregando dados de espectroscopia no UV/Vís e PLS, podem ser utilizados como alternativa na quantificação de misturas de corantes.

Palavras-chave: Corantes; PLS; Espectroscopia, agilidade, alternativa.

\section{Partial least squares regression (PLS) and spectroscopy UV/Vís for quantification of dyes: agility and efficiency for wastewater treatment stations textiles}

\begin{abstract}
The dyes are chemicals with large structural diversity. Have numerous applications, particularly for industries: textiles, food and ceramics. There are several analytical techniques used in these industries for evaluation of these compounds. However, there is a demand for methods where two or more colors are assessed in the same analysis, when such methodologies used can contribute significantly reduced costs, while reducing analysis time and consumption of reagents. Thus, in order diffusion methodologies for simultaneous quantification of dyes, employing the Partial Least Squares Regression (PLS), was used in this work spectroscopy in the UV/Vís associated PLS to build templates for quantification of three colors: orange methyl, methylene blue and congo red. The results indicate that calibration models, using spectroscopic data in the UV/Vis and PLS can be used as an alternative the quantification of mixtures of dyes.
\end{abstract}

Keywords: Dyes; PLS; Spectroscopy, Agility, Alternative. 


\section{Introdução}

O setor têxtil, produz grandes volumes de efluentes, os quais, quando não são destinados corretamente e tratados, podem causar sérios problemas ambientais. $\mathrm{O}$ efluente liberado no processo de tingimento é altamente colorido, e libera uma série de contaminantes devido à presença de corantes, incluindo ácidos, compostos alcalinos, sólidos solúveis e compostos tóxicos que não se fixam nas fibras do tecido durante o processo de tingimento. A remoção desses corantes é um dos grandes desafios para as indústrias de tecidos. Há uma estimativa que cerca de $15 \%$ da produção de corantes são perdidos para o meio ambiente durante a síntese, processamento ou aplicação desses corantes, o que representa um lançamento de cerca de 1,20 toneladas por dia desta classe de compostos para o meio ambiente (Guaratini e Zanoni, 2000; Silva et al., 2009).

Vários problemas são causados devido ao lançamento de efluentes não submetido a tratamentos, dentre estes podemos citar: danos ao abastecimento público, contaminações microbiológicas e produtos químicos, causando alterações na dureza, cor e no sabor da água; o encarecimento do tratamento, comprometimento do abastecimento indústrial, limitação para as indústrias na operação e manutenção das caldeiras, impactos negativos sobre as indústrias de pesca, navegação, agropecuária e na recreação e aumento da mortalidade da fauna e flora aquática. Devido às grandes implicações ambientais é de extrema importância o conhecimento qualitativo e quantitativo dos efluentes gerados.

A regulamentação destes compostos juntamente com a necessidade de tratamentos dos efluentes gerados pelas indústrias, as quais empregam corantes em seus produtos, têm forçado o desenvolvimento de novas metodologias para análises dos resíduos produzidos. Para a identificação dos corantes várias técnicas têm sido empregadas. Dentre eleas pode se mencionar as técnicas voltamétricas de redissolução catódica as quais tem sido aplicado com sucesso para determinação de tartrazina e índigo carmim (Kapor et al., 2001). Também vale mencionar as tecnicas cromatográficas destacando a cromatografia líquida de alta resolução (HPLC) por possibilitarem a determinação qualitativa e quantitativa dos corantes. No caso dos métodos cromatográficos, características como alto custo e envolvimento de várias etapas de extração dificultam a obtenção de uma análise rápida. Neste contexto técnicas espectrofotométricas quando associadas à análise multivariada tem sido uma alternativa com grande potencial (Kapor et al., 2001; Santo e Nagata, 2005; Morgano et al., 2007).

A regressão por mínimos quadrados parciais (PLS) é uma método de calibração multivariada que relaciona uma matriz de dados $\mathbf{X}$ com uma ou mais respostas (matriz Y). PLS é um método bem conhecido da comunidade científica, embora ainda pouco explorado pelas indústrias. A utilização dessas metodologias multivariadas para análise de corantes de interesse das indústrias de alimentos tem se mostrado eficiente (Lopez-de-Alba et al., 1996; Berzas Nevado et al., 1999; Berzas et al., 1999 e Santos e Nagata, 2005).

Diante do exposto, o objetivo deste trabalho foi desenvolver uma metodologia analítica para determinação simultânea dos corantes a qual poderá ser aplicada nos processos de tratamentos de efluentes têxteis, dentre outros. Para tanto, foram utilizados espectros no ultravioleta-visível (UV/Vis) dos corantes alaranjado de metila, azul de metileno e vermelho do congo.

\section{Procedimentos Experimentais}

\subsection{Preparo das soluções}

Foram preparadas soluções dos corantes pela pesagem dos padrões em balança analítica e posterior dissolução e diluição com água destilada, para obtenção das soluções contendo $20 \mu \mathrm{g} \cdot \mathrm{mL}^{-1}$. A partir das soluções-estoque; foram preparadas por diluição com água destilada, as soluções-trabalho contendo os corantes e as misturas destes compostos.

\subsection{Análise multivariada}

Para construção do modelo de regressão multivariada foram utilizados os dados espectrais obtidos em triplicata num espectrofotômetro UV/Vís na região do ultravioleta visível (200-800 $\mathrm{nm})$. 
O método de regressão multivariada utilizado no tratamento dos dados foi o método PLS utilizando o software Pirouette ${ }^{\circledR} 3.11$ (InfoMetrix, Inc., 2003). Este método tem como objetivo encontrar uma relação entre a matriz $(\mathbf{X})$ contendo os espectros das amostras dos corantes do conjunto de calibração e o vetor que armazena as respectivas concentrações dos corantes $(\mathbf{Y})$. O resultado é uma equação semelhante à Equação 1

$$
\mathbf{Y}=\mathbf{X b}+\mathbf{e} \quad \text { Equação } 1
$$

onde b é o vetor de regressão e e o vetor que representa os erros do modelo.

\subsection{Análise estatística}

Os espectros de varredura obtidos na região do UV/Vís para as soluções dos corantes foram divididos em dois subconjuntos: um de validação e outro de calibração. Na determinação do número de variavéis latentes (VL) utilizadas no modelo, é feita uma validação cruzada (validação interna) no conjunto de calibração: uma amostra do conjunto de calibração é excluída, o modelo é construído e, então, estimado a concentração de cada corante. Este processor é repetido até que todas as amostras sejam previstas para " $n$ " variáveis latentes (VL). A determinação do número de VL é uma etapa importante para construção do modelo, pois quanto maior o número no modelo, menor será o desvio da reta de calibração. Entretanto, o aumento de VL também aumenta o ruído e os erros de modelagem (Ferrão e Davanzo, 2005).
Para avaliação do conjunto de amostras de validação usamos os resultados obtidos para o erro quadrático médio de validação (RMSEV). Este critério é útil quando se deseja avaliar se o modelo tende a superdimensionar o número de VLs a serem empregadas (Souza e Ferrão, 2006). Os valores de RMSEV, são calculados com os valores de referência (yi) e os valores estimados com o conjunto de validação, conforme equação 2 .

$$
R M S E V=\sqrt{\frac{\sum_{i=l}^{n}\left(y_{i}-\hat{y_{i}}\right)^{2}}{n}}
$$

\section{Equação 2}

Os coeficientes de correlação $\left(\mathrm{R}^{2}\right)$ entre os valores das concentrações predito pelo modelo utilizando espectros UV/Vís e os valores referência das amostras do conjunto de calibração e de validação também foram avaliados.

\section{Resultados e discussão}

As estruturas químicas dos corantes utilizados para construção dos modelos são apresentadas na Figura 1. Os corantes alaranjado de metila (Figura 1a) e vermelho do congo (Figura 1c) são pertencentes à classe dos azos corantes. Estes compostos são extensivamente utilizados nos processos das indústrias têxteis, sendo caracterizados por elevada resistência frente a processos aeróbios de biodegradação o que acarreta persistência nos processos convencionais de tratamento de resíduos (Souza et al., 2006).

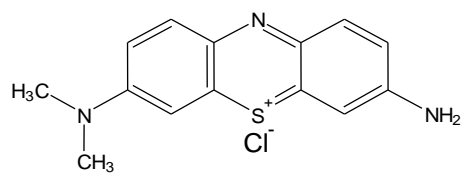

b

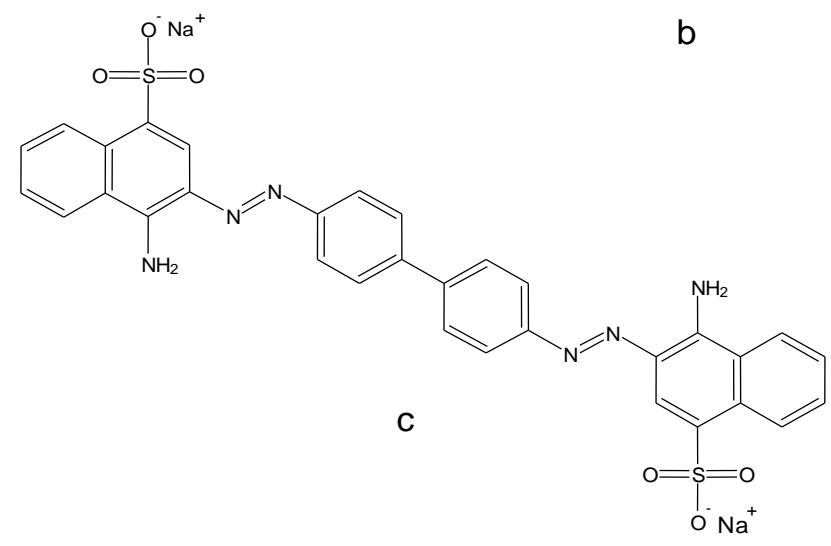

Figura 1. Estruturas dos corantes alaranjado de metila (a), azul de metileno (b) e vermelho do congo (c). 
A faixa de concentração estudada para construção do modelo variou entre 2,0 e 20,0 $\mu \mathrm{g} \cdot \mathrm{mL}^{-1}$ para os corantes isolados ou a mistura destes compostos. A Figura 2 apresenta o conjunto de espectros UV-Vis obtidos a partir das soluções padrão dos corantes estudados. Observa-se as bandas de absorção em $460 \mathrm{~nm}$ característico do alaranjado de metila (Figura 2A), no comprimento de onda de $665 \mathrm{~nm}$ correspondente a absorção máxima do azul de metileno (Figura 2B) e no comprimento de $510 \mathrm{~nm}$ correspondente ao vermelho do congo (Figura 2C).

Os modelos para quantificação dos corantes foram desenvolvidos considerando-se toda a região espectral (200 a $800 \mathrm{~nm}$ ) Figura 2, utilizando-se
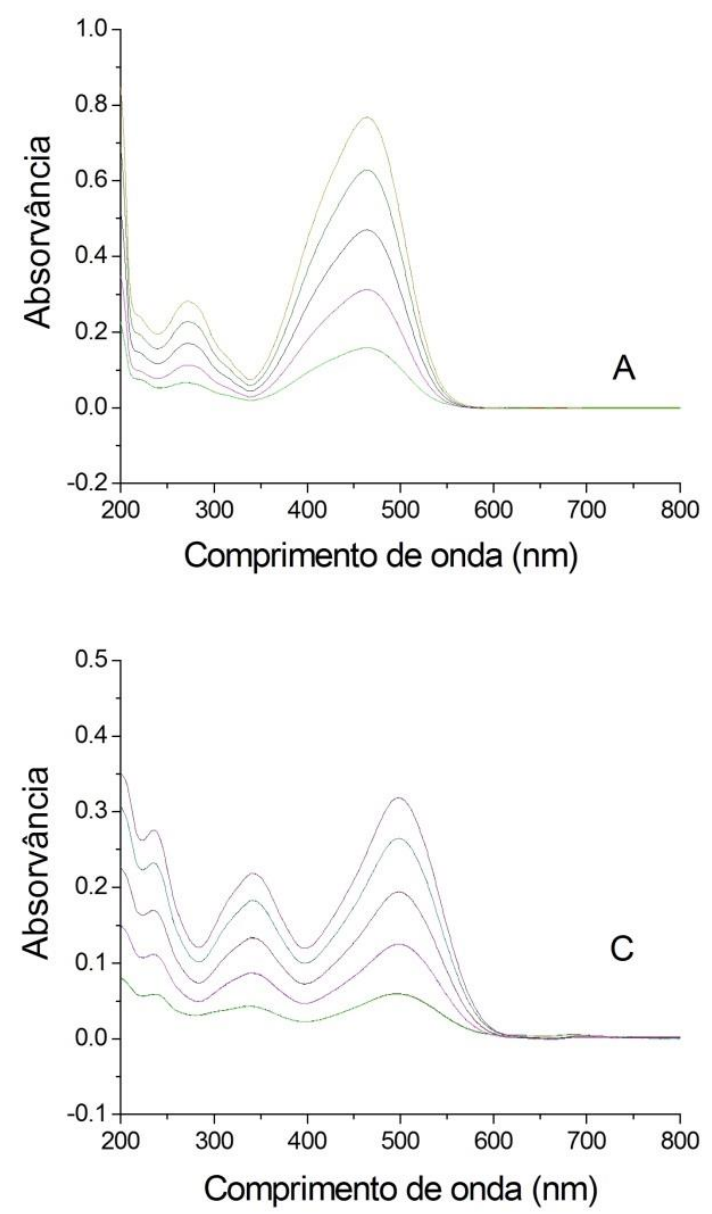

dados espectrais na sua forma original, centrados na média e o procedimento de validação cruzada. A seleção do número de variáveis latentes foi realizada após a análise do gráfico do erro médio quadrático de previsão para o conjunto de validação (RMSEV) pelo número de variáveis latentes (Figura 3).

Pode se observar na Figura 3 o comportamento do REMSEV para o conjunto de validação em função do número de variáveis latentes utilizados nos modelos. O valor mínimo do REMSEV exibido pelo conjunto de validação é atingido quando se utiliza um número de variáveis latentes igual a 4.
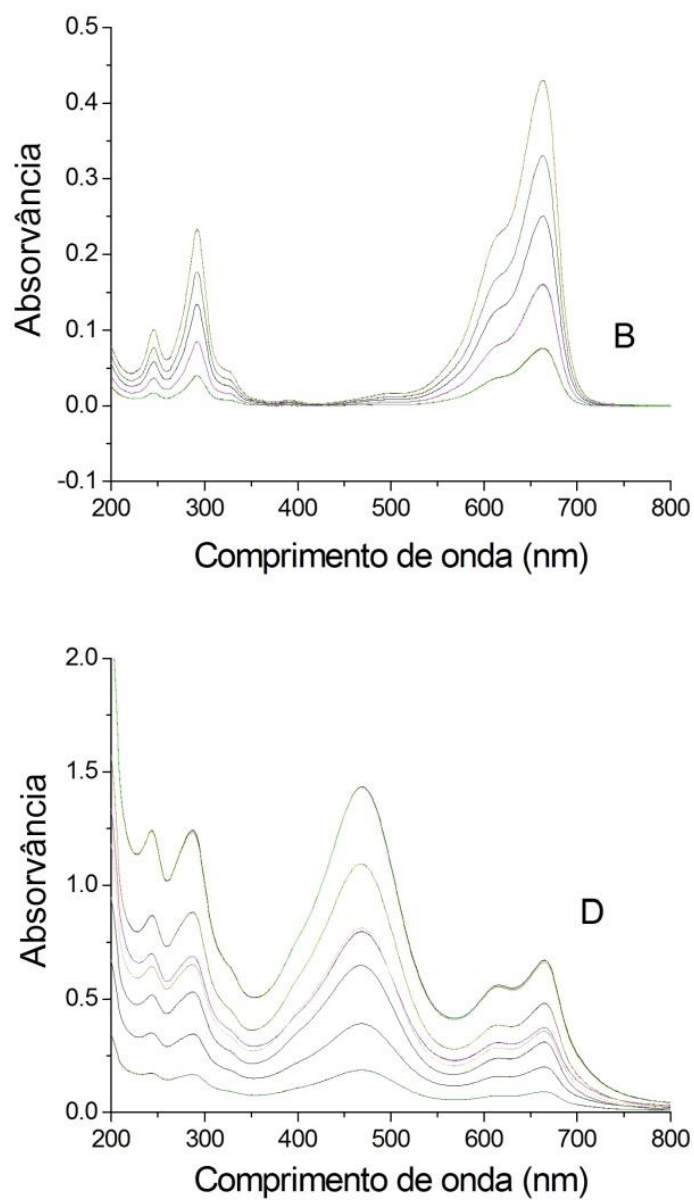

Figura 2. Espectros de absorção em diferentes concentrações, dos corantes alaranjado de metila (A), azul de metileno (B), vermelho do congo (C) e da mistura destes corantes (D). 


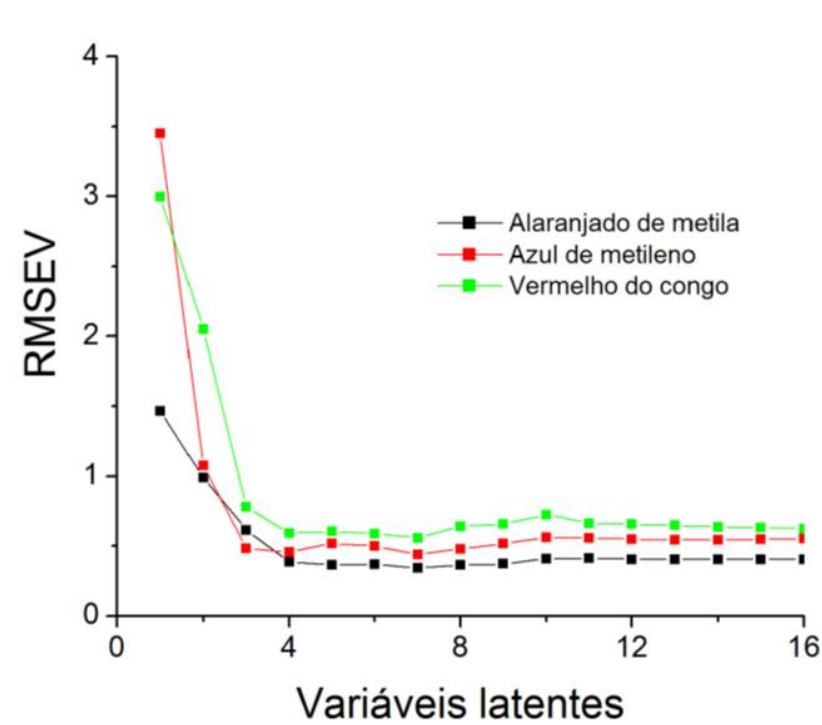

Figura 3. Gráfico de RMSEV versus variáveis latentes

Os resultados da calibração e validação são apresentados na Figura 4. Esta figura exibe os valores experimentais versus os valores preditos pelo modelo PLS com 4 variáveis latentes. A capacidade de previsão dos modelos para a concentração dos corantes, visualizadas na Figura 4, podem ser avaliadas comparando as concentrações previstas com os valores de referência, os quais são muito próximos $\left(\mathrm{R}^{2}>0,99\right.$ para ambos corantes). Também é possivel observar que não foram constatadas a presença de amostras anômalas, e todos os padrões utilizados apresentaram-se dentro dos limites aceitáveis.
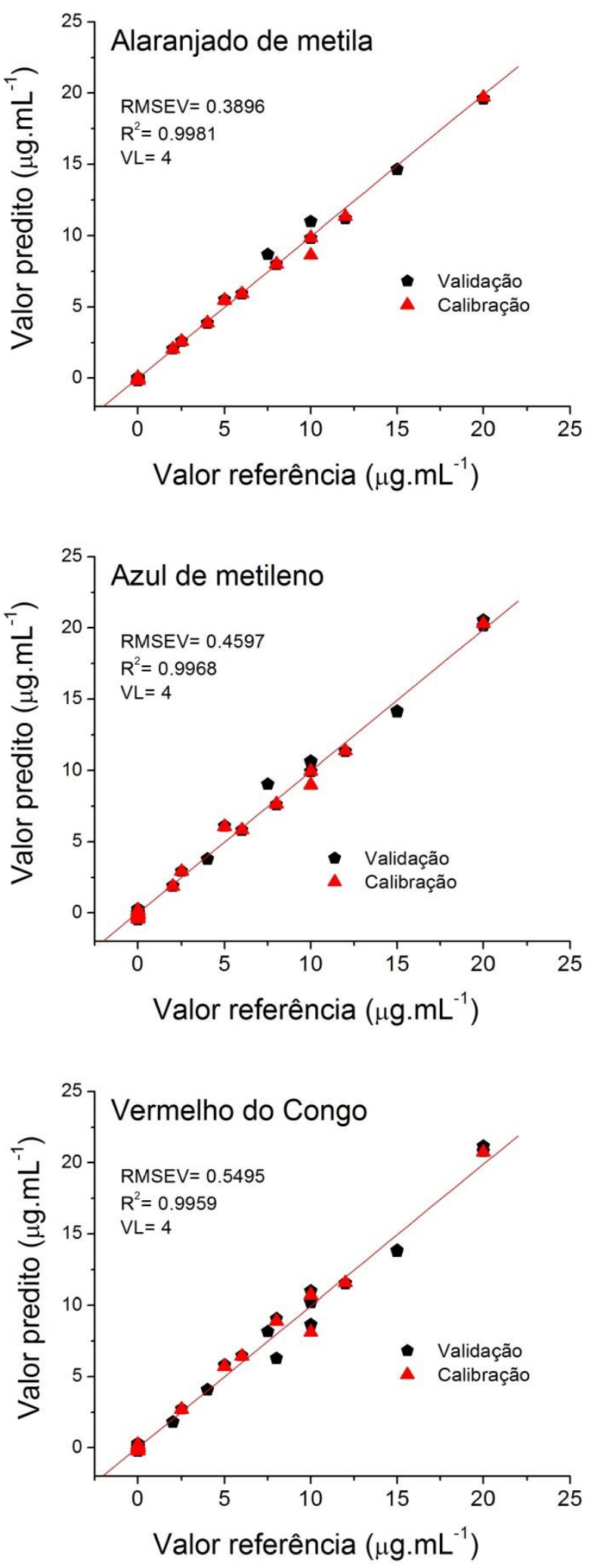

Figura 4. Valores reais versus valores previstos pelo modelo PLS para as concentrações dos corantes. 


\section{Conclusão}

A espectroscopia no UV/Vís associada com o método de calibração multivariada (PLS) é uma técnica fácil e rápida para quantificação simultânea de corantes. Os modelos de regressão construídos podem ser aplicados em vários setores, destacando aqueles envolvidos nos processos de tratamentos de efluentes têxteis. Dentre as vantagens dos métodos estudados é importante destacar a redução do tempo de análise, pouca manipulação das amostras, diminuição de resíduos químicos e redução do custo de análise.

\section{Referências}

BERZAS NEVADO, J. J.; GUIBERTEAU CABANILLAS, C.; CONTENTO SALCEDO , A. M. Spectrophotometric determination of three food dyes by using the first derivative of ratio spectra. Talanta, v. 42, p. 2043-2051, 1995.

BERZAS NEVADO, J. J.; RODRÍGUES FLORES, J.; VILLASEÑOR LLERENA, M.J.; RODRÍGUEZ FARIÑAS, N. Simultaneous spectrophotometric determination of tartrazine, patent blue $\mathrm{V}$, and indigo carmine in commercial products by partial least squares and principal component regression methods. Talanta, v. 48, p. 895-903, 1999.

FERRÃO, M. F.; DAVANZO; C. U. Horizontal attenuated total reflection applied to simultaneous determination of ash and protein contents in commercial wheat flour. Analytica Chimica Acta, v. 440, p. 411-415, 2005.

GUARATINI, C. C. I.; ZANONI, M. V. B. Corantes têxteis. Química Nova, v. 23,p. 71-78, 2000.

INFOMETRIX Inc. Pirouette $\AA$ for Windows, version 3.11, Woodinville, WA, USA, 2003.

KAPOR, M. A.; YAMANAKA, H.; CARNEIRO, P. A.; ZANONI, M. V. B. Eletroanálise de corantes alimentícios: determinação de índigo carmim e tartrazina. Eclética Química, v. 26, p. 53-68, 2001.

LOPEZ-DE-ALBA, P. L.; WRÓBELKACZMARCZYK, K.; WRÓBEL , K.; LÓPEZMARTINEZ, L ; AMADOR HERNÁNDEZ, J. Spectrophotometric determination of Allura Red (R40) in soft drink powders using the universal calibration matrix for partial least squares multivariate method. Analytica Chimica Acta, v. 330, p. 19-29, 1996.

MORGANO, M. A.; FARIA, C. G; FERRAO, M. F.; FERREIRA, M. M. C. Determinação de açúcar total em café cru por espectroscopia no infravermelho próximo e regressão por mínimos quadrados parciais. Química Nova, v. 30, p. 346350, 2007.

SANTOS, M.E; NAGATA, N. Determinação espectrofotométrica simultânea de corante amarelo tartrazina e amarelo crepúsculo via regressão por componentes principais. UEPG: Ciências Exatas e da Terra, Ciências Agrárias e Engenharias, v. 11, p. 51-60, 2005.

SILVA, A. C., OLIVEIRA, D. Q. L., OLIVEIRA, L. C. A., ANASTÁCIO, A. S., RAMALHO, T. C., LOPES, J. H., CARVALHO, H. W. C., TORRES, C. E. R., Nb-containing hematites $\mathrm{Fe}_{2-\mathrm{x}} \mathrm{Nb}_{\mathrm{x}} \mathrm{O}_{3}$ : The role of $\mathrm{Nb5}+$ on the reactivity in presence of $\mathrm{H}_{2} \mathrm{O}_{2}$ or ultraviolet light. Applied catalysis A : General, v. 357, p. 79-84, 2009.

SOUZA, J. S.; FERRAO, M. F. Aplicações da espectroscopia no infravermelho no controle de qualidade de medicamentos contendo diclofenaco de potássio. Parte I: dosagem por regressão multivariada. Revista Brasileira de Ciencias Farmaceuticas, v. 42, p. 437-445,2006.

SOUZA, C. L.; PERALTA-ZAMORA, P. Degradação redutiva de azo-corantes utilizando-se ferro metálico. Engenharia Sanitaria e Ambiental, v. 11, n. 1, p. 16-20, 2006.

Artigo selecionado entre os 10 melhores do VIII Encontro Mineiro de Engenharia de Produção - EMEPRO 2012. 